CARNETS DE Carnets de géographes

GÉOGRAPHES.

$14 \mid 2020$

Varia

\title{
Abstraction, production sociale et production de l'espace
}

Diego Scalco

\section{(2) OpenEdition}

Journals

Édition électronique

URL : https://journals.openedition.org/cdg/6537

DOI : $10.4000 /$ cdg. 6537

ISSN : 2107-7266

Éditeur

UMR 245 - CESSMA

Référence électronique

Diego Scalco, "Abstraction, production sociale et production de l'espace », Carnets de géographes [En ligne], 14 | 2020, mis en ligne le 15 décembre 2020, consulté le 20 mai 2021. URL : http:// journals.openedition.org/cdg/6537; DOI : https://doi.org/10.4000/cdg.6537

Ce document a été généré automatiquement le 20 mai 2021.

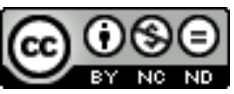

La revue Carnets de géographes est mise à disposition selon les termes de la Licence Creative Commons Attribution - Pas d'Utilisation Commerciale - Pas de Modification 4.0 International. 


\title{
Abstraction, production sociale et production de l'espace
}

\author{
Diego Scalco
}

\section{Deux régimes d'abstraction}

1 Comment parvenir à une problématisation de l'urbain sur la base d'interrogations relevant de cadres aussi éloignés, du moins en apparence, que ceux de la sociologie formelle, de la planification moderne, de la sculpture processuelle, de l'anarchitecture et de la géographie matérialiste ? Le défi méthodologique relevé ici consiste à ménager des transitions ponctuelles, vouées moins à faire éclater les découpages disciplinaires en vigueur qu'à dégager le plan de clivage entre les démarches concernées. Au risque d'anticiper sur le contenu des analyses à mener, force est de signaler que ce plan est idéologiquement orienté, parfois de façon latente, et qu'en conséquence il excède les diverses théories et pratiques prises dans leurs singularités comme dans leurs contingences. Notre argumentation s'étaye donc sur la différence, voire sur l'antagonisme, entre deux conceptions fondamentales de la relation réciproque que la socialisation entretient avec la spatialisation. Différence qui, dans le titre, est délinéée par l'emprunt, respectivement à Georg Simmel (1858-1918) et à Henri Lefebvre (1901-1991), des formules «production sociale» (Simmel, 1981: 93) et «production de l'espace » (Lefebvre, 2000).

Dans l'objectif d'articuler la « problématique de la ville» (Lefebvre, 2009 : 39), Lefebvre distingue, entre autres éléments de réflexion, «l'urbanisme comme doctrine, c'est-àdire comme idéologie, interprétant les connaissances partielles, justifiant les applications, les élevant (par extrapolation) à une totalité mal fondée ou mal légitimée » (ibid.: 39). Sa distinction sert dorénavant d'hypothèse de travail, pour autant qu'elle pointe non seulement ce qu'il y a de sociologique dans certaines formes d'urbanisme, mais aussi les principes que l'urbanisme développé au fil des projets de Le Corbusier (Charles-Édouard Jeanneret-Gris, 1887-1965) partage avec la sociologie de Simmel ${ }^{1}$. En considération de l'ascendant qu'il exerce sur ses contemporains, y compris 
sur un architecte aux convictions socialistes comme Ludwig K. Hilberseimer (1885-1967), Le Corbusier fait figure de parangon international de l'urbaniste qui, selon les termes de Lefebvre, s'attribuerait «la capacité de concevoir un espace social harmonieux, normal et normalisant " (ibid.: 41) dans le dessein "d'accorder à cet espace (qui se trouve comme par hasard identique à l'espace des géomètres, celui des topologies abstraites) les réalités sociales préexistantes » (ibid.: 42). Il reste à déterminer dans quelle mesure et sous quels aspects les modes présumés d'un tel accord ou, plutôt, d'une telle intégration socio-spatiale, sont assimilables à ceux de la production sociale au sens de Simmel, modelée, elle aussi, sur la géométrie.

3 Ces remarques introductives motivent le recours au concept d'abstraction, en référence notamment aux topologies urbanistiques évoquées par Lefebvre, aux typologies sociologiques de matrice simmelienne, ainsi qu'aux démarches théoriques et pratiques qui y aboutissent. Notre critique de l'abstraction va se développer, de manière quelque peu inattendue, par l'intermédiaire de l'abstrait lui-même. La méthode d'analyse adoptée dorénavant dans le sillage de Lefebvre, parmi d'autres auteurs, dérive effectivement de la conception de l'abstrait spécifique à la pensée de Karl Marx (1818-1883). Conception tout aussi clivée que celle dessinée en creux par la différence, en matière d'intervention sur l'espace métropolitain, entre l'urbanisme planifié de Le Corbusier ou de Hilberseimer d'une part, et la sculpture processuelle de Richard Serra (né en 1939) ou l'anarchitecture de Gordon Matta-Clark (1943-1978) d'autre part. À la différence de l'abstraction à partir du concret, « la méthode qui consiste à s'élever de l'abstrait au concret n'est que la manière pour la pensée de s'approprier le concret » (Marx, 1980: 35). Et le matérialisme paradoxal, pour ainsi dire, des démarches abstraites de Serra et de Matta-Clark réside, comme il s'agit de le montrer, en ceci qu'elles mettent simultanément au jour et en cause le substrat idéologique de la planification moderne. Le choix des auteurs, des architectes et des artistes mentionnés s'explique alors par l'opportunité de retracer quelques inflexions majeures subies par l'abstraction durant le siècle dernier, au moyen de l'analyse contrastive de réflexions, de projets et d'interventions qui, nous semble-t-il, en sont particulièrement représentatifs.

\section{Des modes présumés d'intégration socio-spatiale}

4 Dans l'esprit de certains urbanistes du début du siècle dernier, le plan constitue un mode de représentation permettant d'appréhender la complexité du réel en transparence et, plus précisément, de saisir dans un état de superposition plusieurs orientations et situations spatiales. Ainsi comprise, la planification rejoint la cartographie qui, la première, se distingue par la réduction du référent à la coordonnée et de l'espace à la grille. Ces disciplines postulent l'adéquation de la représentation au représenté, qu'il soit question d'objets spécifiques, comme dans la carte, ou génériques, comme dans le plan. En cartographie, les déterminations territoriales sont synthétisées par abstraction, ce qui en retour détermine la manière dont le territoire lui-même est parcouru et, éventuellement, aménagé. Il en va de façon analogue pour la planification telle que l'entendent Le Corbusier et Hilberseimer au cours des années 1920. Les conceptions de l'urbanisme prônées par ces architectes suscitent tout de même un intérêt particulier étant donné que, grâce au recul désormais possible, leur comparaison décèle une idéologie largement partagée dans la première moitié du $\mathrm{xx}^{\mathrm{e}}$ 
siècle en dépit des multiples orientations politiques. Hilberseimer s'attèle certes à la théorisation comme à la planification de la métropole socialiste, mais il ne parvient pas non plus à se déprendre d'une vision productiviste de l'intégration socio-spatiale ${ }^{2}$. La plasticité d'une telle idéologie confère alors tout leur sens aux développements subséquents.

Chez Le Corbusier ou Hilberseimer, le plan se rapporte tant à la représentation de l'espace qu'aux modes concrets d'organisation et de transformation des comportements qui y prennent place. En d'autres termes, les relations géométriquement établies dans le cadre du plan reflètent la composition sociale de la métropole et, en retour, tendent à devenir des formes de recomposition par le haut. Ce processus d'autonomisation tient au fait qu'il est possible d'intégrer dans le plan tous les facteurs de la planification, y compris sous le rapport sociétal. Les dispositifs urbains qui s'élaborent sous les crayons respectifs de Le Corbusier et de Hilberseimer sont censés entretenir une relation utopique avec la métropole actuelle, dans la mesure où ils visent à disloquer l'imaginaire métropolitain. Toutes les déterminations spatiales et sociales de cet imaginaire y sont pourtant contenues, et cela en conformité, mutatis mutandis, avec l'idée de Simmel selon laquelle les structures de la socialisation seraient permanentes et déterminables indépendamment des circonstances. La méthode déductive de Simmel constitue à son tour une abstraction d'ordre typologique, sinon immédiatement topologique, effectuée en fonction de la distinction entre les contenus et les formes sociales :

La socialisation est donc la forme, aux réalisations innombrables et diverses, dans laquelle les individus constituent une unité fondée sur ces intérêts - matériels ou idéaux, momentanés ou durables, conscients ou inconscients, agissant comme des causes motrices ou des aspirations téléologiques - et à l'intérieur de laquelle ces intérêts se réalisent. Dans tout phénomène social existant, le contenu et la forme sociale constituent une réalité concrète unitaire; une forme sociale ne peut pas plus acquérir une existence détachée de tout contenu qu'une forme spatiale ne peut exister sans la matière dont elle est la forme (Simmel, 1999 : 44).

Paradoxalement, la reconnaissance de la genèse sociale des catégories logiques et empiriques n'empêche pas Simmel de leur attribuer un caractère a priori en vertu duquel elles régiraient la connaissance et l'expérience concrètes. Il ne s'agit pas des catégories kantiennes, puisque la sociologie simmelienne requiert «la prise de conscience du mode de production sociale qui s'est glissé entre la production purement individuelle et la production transcendante» (Simmel, 1981: 93), et pourtant elles remplissent une fonction comparable en tant que conditions de possibilité de la socialisation ${ }^{3}$. Simmel est ainsi conduit à établir un parallèle méthodologique entre la sociologie et la géométrie :

On pourrait aussi saisir ce qui est vraiment «société » dans la société de la même façon que la géométrie détermine ce qui est vraiment spatial dans les choses spatiales. La sociologie, comme théorie de l'être-société de l'humanité, qui peut aussi être un objet de science à d'innombrables points de vue, a donc avec les autres sciences particulières le même rapport que la géométrie avec les sciences physiques et chimiques de la matière : elle considère la forme par laquelle la matière devient un corps empirique - forme qui bien entendu n'existe pour elle-même que dans l'abstraction, exactement comme les formes de la socialisation (Simmel, 1999 : 49).

7 Il s'infère de ce parallèle que la déduction des catégories a priori que sont l'individualité, le rôle et la structure peut s'inverser en induction, les formes de 
socialisation dégagées par Simmel risquant constamment d'être prises aussi pour des modes efficaces de planification et, surtout, d'intégration socio-spatiale.

\section{Cartographie sociale et géométrisation de l'espace}

8 Une fois perçue comme une cartographie sociale, l'approche sociologique d'inspiration simmelienne tend à converger avec la géométrisation subie par l'architecture et l'urbanisme dans le passage de la ville à la métropole. Selon Simmel, l'individu est porté à la socialisation, mais son individualité demeure irréductible à la dimension sociale puisqu'il maintient toujours un certain degré d'autonomie ${ }^{4}$. La constitution d'une telle dimension implique « de désigner l'homme en fonction d'un type implicite, avec lequel ne coïncide pas son pur être-pour-soi » (ibid. : 69). Les actions individuelles ne cessent alors de se réintégrer à ce qui, en dernière instance, découle d'une abstraction, à savoir la société. Le véhicule de leur réintégration est le rôle que l'individu assume ou se voit assigné dans les différents groupes, "et cette condition inévitable, tout à fait automatique, est, dans notre représentation de l'autre, l'un des moyens de donner à sa personnalité et à sa réalité la qualité et la forme requises par sa sociabilité » (ibid. : 70). L'inégalité entre les individus à l'intérieur d'un groupe étant corrélée à l'inégalité entre les groupes à l'intérieur de la structure, Simmel en vient à concevoir la société comme un « ordre » (kosmos, en grec ancien) à l'équilibre tantôt statique, tantôt dynamique :

Mais si l'inégalité des éléments fait apparaître toutes les relations ou toutes les qualités à l'intérieur de cet ordre comme caractérisées individuellement, avec des places clairement établies, alors la société apparaît comme un cosmos, avec une infinie diversité d'êtres et de mouvements, certes, mais où chaque point ne peut exister ou évoluer que de cette manière déterminée si l'on veut éviter que la structure de l'ensemble soit modifiée (ibid. : 76).

Simmel caractérise le fonctionnement du cosmos social par l'exemple de la bureaucratie $e^{5}$ nous permettant ainsi d'établir un lien avec l'urbanisme de Le Corbusier. Ville contemporaine de trois millions d'habitants ${ }^{6}$ est, en effet, un projet pour le centre administratif d'une société industrielle avancée. À l'instar de la réflexion de Simmel, la planification corbuséenne est directement concernée par la transition de la ville à la métropole en tant que conséquence de l'industrialisation intensive. La modernité, s'il en est, de son urbanisme se mesure à l'aune de la métropole en tant que résultante de la division du travail et se définit initialement par rapport à la vision de la ville promue par Camillo Sitte (1843-1903) au tournant du xixe siècle. Dans son interprétation de la ville d'art, Sitte critique le présupposé selon lequel la question de la vitesse de circulation serait fondamentale en urbanisme et va jusqu'à assimiler l'aménagement orthogonal à la négation de toute qualité esthétique ${ }^{7}$. Chez Le Corbusier, la géométrisation et l'enrégimentation du mouvement constituent les paramètres principaux d'une doctrine à visée utopique, aux antipodes de l'interprétation de Sitte qui se fonde sur la dissociation des problèmes techniques et formels. L'architecte franco-suisse voit néanmoins dans le plan une table rase sur laquelle s'élèvent les édifices singuliers et procède à la mise en scène de l'émergence architecturale, laquelle se trouve rehaussée par la disproportion entre les vieilles et les nouvelles constructions. En témoigne le Plan Voisin ${ }^{8}$ pour le réaménagement du centre de Paris, où les quelques bâtiments et monuments historiques préservés se trouvent rapetissés par comparaison avec les tours de la cité administrative. Ces caractéristiques se 
trouvent déjà dans Ville contemporaine de trois millions d'habitants, projet fournissant les linéaments de la doctrine :

(La ville dessinée ici est un pur jeu de conséquences géométriques.) Un nouveau module vaste (400 mètres) l'anime toute. Le quadrillage régulier de ses rues recoupées à 400 mètres et à 200 mètres est uniforme (orientation facile du voyageur), mais il n'est pas un de ses aspects qui soit semblable à un autre. Ici jouent, en symphonie fuguée, les forces de la géométrie (Le Corbusier, 1925 : 167-178).

10 Le fait que ce plan recoupe l'organisation fonctionnelle de la métropole capitaliste n'exclut pas, aux yeux de Le Corbusier, que la hiérarchie concentrique mise en place puisse devenir aussi un facteur de production sociale dans l'acception discutée plus haut, vu que le partage géométrique de l'espace s'opère suivant les trois catégories $a$ priori de l'individualité, du rôle et de la structure. À cela s'ajoute l'idéal saint-simonien d'une union entre l'art et l'industrie ${ }^{9}$. En toute logique, l'évolution du standard (ou standart, selon la terminologie corbuséenne) est reliée à la concurrence, suivant un schéma d'explication évolutionniste :

Le Parthénon est un produit de sélection appliquée à un standart établi. Depuis un siècle déjà, le temple grec était organisé dans tous ses éléments. Lorsqu'un standart est établi, le jeu de la concurrence immédiate et violente s'exerce. C'est le match ; pour gagner, il faut faire mieux que l'adversaire dans toutes les parties, dans la ligne d'ensemble et dans tous les détails. C'est alors l'étude poussée des parties. Progrès. Le standart est une nécessité d'ordre apporté dans le travail humain. Le standart s'établit sur des bases certaines, non pas arbitrairement, mais avec la sécurité des choses motivées et d'une logique contrôlée par l'analyse et l'expérimentation (Le Corbusier, 1928 : 106-108).

11 Dans cette perspective, il en va de la métropole comme du temple grec, les éléments de l'urbanisme étant fixés au préalable et l'enjeu se résumant, par conséquent, à en proposer une synthèse adaptée à la société industrielle. Ainsi, dans Ville contemporaine de trois millions d'habitants, cette synthèse connaît une genèse par le haut, effet de l'anticipation rationnelle :

L'architecture agit sur des standarts. Les standarts sont choses de logique, d'analyse, de scrupuleuse étude. Les standarts s'établissent sur un problème bien posé. L'architecture est invention plastique, est spéculation intellectuelle, est mathématique supérieure. Le standart, imposé par la loi de sélection, est une nécessité économique et sociale. L'harmonie est un état de concordance avec les normes de notre univers (ibid. : 115).

12 La métropole imaginée par Le Corbusier se réduit à une configuration accomplie au niveau d'une structure unique, à la fois abstraite de la multiplicité des phénomènes socio-spatiaux et surimposée à cette même multiplicité. L'actuel et le possible peuvent ainsi coexister dans et par les rapports d'équivalence entre les orthogonales au sein du plan en tant que dispositif, comme si l'étendue des situations réelles et éventuelles était révélée au bout de la rationalisation la plus rigoureuse.

\section{De l'utopie à l'atopie}

13 Il est question, pour Le Corbusier, « d'arriver en construisant un édifice théorique rigoureux, à formuler des principes fondamentaux d'urbanisme moderne " (Le Corbusier, 1925 : 158). Le plan est à l'urbanisme ce que la carte est à la géographie, il doit lui aussi fixer les conditions de possibilité de toute idéation ou réalisation urbanistique efficace. Et ces 
conditions sont géométrico-mathématiques, sinon métaphysiques, d'où la référence explicite à Platon (environ 428-348 avant notre ère). «L'ARCHITECTURE, c'est l'art par excellence, qui atteint à l'état de grandeur platonicienne, ordre mathématique, spéculation, perception de l'harmonie par les rapports émouvants. Voilà la FIN de l'architecture » (Le Corbusier 1928 : 86-87), assure Le Corbusier. L'urbanisme, quant à lui, est la « formulation qu'il donna à ses espoirs saint-simoniens d'une Europe au-delà de la politique, débarrassée des barrières idéologiques ou nationales, unie par la logique objective de la technologie et de la géographie » (Fishman, 1979 : 192).

La réflexion sur l'urbain menée par Hilberseimer fait également état de l'internationalisation du phénomène métropolitain, ainsi que des problèmes méthodologiques que la planification est appelée à pallier ${ }^{10}$. Les affinités indéniables entre son Projet de développement de Berlin ${ }^{11}$ et le Plan Voisin de Le Corbusier ne nous dispensent pas pour autant de considérer les spécificités de l'utopie urbanistique qu'il propose et de la critique qu'il en fait rétrospectivement. Persuadé que seulement dans " une société socialement ordonnée, où la production pourvoit aux besoins des hommes et non pas à la cupidité d'individus privilégiés, la métropole devient un organisme sensé » (Hilberseimer, 2012: 89), l'architecte allemand arrive à des conclusions radicales. Dans le projet en question, « [1]a règle générale, la loi, est exaltée et mise en évidence, l'exception est laissée de côté, la nuance est éradiquée, la masse domine, le chaos est forcé de devenir forme logique, sans ambiguïté, mathématique, prescriptive " (ibid.: 280). Il ressort de ces propos que les variations opérées sur la structure géométrique doivent engager une transformation des interactions spatiales impliquant, à son tour, une transformation de la conscience sociale. Et cela conformément à la conviction que «l'abstraction par rapport au cas spécifique est en mesure de montrer comment les éléments disparates qui constituent la métropole peuvent être intégrés dans un ordre de relations denses " (ibid. : 112).

Le constat que "les sociétés produisent des formes d'organisation correspondantes à leurs capacités productives respectives " (ibid. : 84) conduit Hilberseimer à reconnaître que «la forme actuelle de la métropole correspond essentiellement à la forme économique de l'impérialisme capitaliste, dont le pouvoir se développe grâce au concours de la science et des technologies de production et s'exerce avec une intensité croissante non seulement sur l'économie mondiale, mais aussi nationale " (ibid.: 86). Dès lors, Hilberseimer entreprend de planifier une alternative socialiste à l'urbanisme corbuséen qui, d'après lui, n'est pas neutre mais idéologiquement modelé sur l'assise capitalistique. Son projet de Ville verticale ${ }^{12}$ vise à subvertir la métropole "comme création du grand capital tout-puissant, comme empreinte de son anonymat, comme modèle urbain régi par des principes socioéconomiques et psychosociaux particuliers, qui permet simultanément l'isolement maximal et l'intégration la plus stricte de ses habitants " (ibid. : 86). L'équilibre instauré dans le cadre de ce plan est censé préfigurer l'organisation à venir, la construction de nouvelles relations spatiales étant téléologiquement orientée vers l'abolition des contradictions dans la sphère sociopolitique et urbanistique. Cependant, dans ce cas aussi, l'élément modulaire est subsumé sous la structure géométrique en fonction de sa situation, tout comme l'individu est censé être assimilé par la structure sociétale en fonction de son rôle. Autant dire que l'alternative socialiste recherchée par Hilberseimer ne met pas en doute les trois catégories a priori qui, chez Simmel, constituent les conditions de 
possibilité de la production sociale. Il est révélateur, à ce sujet, que Hilberseimer finisse par renier son propre projet :

La répétition des blocs entraîne une trop grande uniformité. Tous les éléments naturels sont exclus : aucun arbre, aucune zone herbeuse ne rompt la monotonie. Le résultat est une nécropole, plutôt qu'une métropole, un paysage stérile d'asphalte et de ciment, inhumain sous tous les aspects (Hilberseimer, 1988 : 17).

La contradiction sous-jacente tient, une fois de plus, à la coexistence de deux aspects, l'un heuristique et l'autre productif, qui en définitive demeurent inconciliables. L'utopie urbanistique élaborée par Hilberseimer tend, comme celle de Le Corbusier, à s'inverser en atopie, en un non-lieu abstrait, corroborant ainsi la tendance que Françoise Choay appelle "réduction sémantique» (Choay, 1972: 22) de l'espace métropolitain. Toujours selon elle, «[1]e caractère abstrait de l'espace urbain actuel est imputable à ce dernier [métalangage] plus qu'à un rôle privilégié attribué au sens de la vue après le $\mathrm{xv}^{\mathrm{e}}$ siècle » (ibid. : 24). La morphologie picturale de la ville d'art sous-tend l'urbanisme préconisé par Sitte, plutôt que l'aménagement orthogonal de la métropole capitaliste. Et Choay de signaler «le remplacement progressif du "langage de la ville" par un langage sur la ville» (ibid. : 28), voire « la constitution d'un discours autonome sur l'urbain [...] qui, du même coup, pose son objet - la ville - et devient un facteur de sa morphogénèse " (ibid. : 28-29). Remplacement et constitution qui accomplissent la transformation des prémisses en conséquences, que ce soit sous le rapport spatial ou social. Chez Le Corbusier ou Hilberseimer, les procédés axonométrique et isométrique servent à instituer l'isotopie et l'isonomie à large échelle. Cette opération est envisagée comme un facteur de morphogénèse des situations socio-spatiales, sous prétexte qu'elle abstrait les conditions intrinsèques, nécessaires, de la spatialisation et de la socialisation, tandis qu'elle n'abstrait que les conditions qui leur sont actuellement imposées de l'extérieur par les facteurs économiques.

17 À ce stade de notre réflexion, il est compréhensible que, même s'ils demeurent irréalisés, les projets de Le Corbusier et de Hilberseimer confirment, chacun selon ses particularités, l'idéologie encore à l'œuvre dans la planification actuelle. L'inversion de l'utopie en atopie obéit à la logique implicite en la poussant à son paroxysme, c'est-àdire à son degré d'abstraction maximal. Telle qu'elle vient de se définir, l'atopie n'a donc rien d'hétérotopique au sens de Michel Foucault (1926-1984), puisqu'elle correspond à l'état des lieux. Le concept foucaldien d'hétérotopie permet par contre d'introduire et de relier les interventions sur l'espace métropolitain auxquelles procèdent Serra et Matta-Clark. En l'occurrence, l'effet concret que visent la sculpture processuelle et l'anarchitecture consiste, par-delà leurs différences, en la production de " contre-emplacements " (Foucault, $2004:$ 15). Foncièrement hétérogènes, " elles ont pour rôle de créer un espace d'illusion qui dénonce comme plus illusoire encore tout l'espace réel, tous les emplacements à l'intérieur desquels la vie humaine est cloisonnée » (ibid. : 18). Il est d'ailleurs significatif que ce soit par des moyens abstraits qu'elles parviennent à critiquer le double caractère abstractif et prospectif de l'urbanisme moderne. Chez Serra, la critique est véhiculée par le contraste qui se produit entre la sculpture et son site d'installation en tant que support idéologique de comportements planifiés. Chez Matta-Clark, elle porte sur les transformations économiques suite auxquelles certaines aires urbaines sont vouées à l'abandon et au dépérissement progressifs.

18 Les réalisations autour desquelles s'articule le paragraphe successif sont donc symptomatiques de la crise que traverse l'urbanisme planifié depuis le tournant des 
années 1950. Selon Franco Farinelli (né en 1948), «les modèles urbains mis au point entre les deux guerres sont encore topographiques parce que la ville imite encore la carte, parce que son fonctionnement obéit encore à sa logique " (Farinelli, $2009: 201$ ). Topographiques, certes, mais surtout atopiques au sens défini supra, comme l'atteste l'accueil favorable réservé aux idées de Le Corbusier. Manfredo Tafuri (1935-1994) observe que « l'idéologie du plan est dépassée par la réalité du plan, dès lors que celui-ci quitte le niveau de l'utopie pour devenir un mécanisme opératoire efficace » (Tafuri, 1979 : 117). La planification et la cartographie se recoupent encore une fois en ceci que toutes deux informent l'espace concret à mesure qu'elles s'en autonomisent. Et l'espace concrètement modelé sur ce processus d'autonomisation acquiert lui-même une réflexivité autotélique en vertu de laquelle il peut se reproduire aussi indéfiniment qu'abstraitement. À l'encontre de cette situation, les démarches de Serra et de MattaClark entraînent un décalage par rapport aux abstractions socio-spatiales courantes. Elles en disloquent la «structure anthropo-géographique» (ibid.: 105-106), pour reprendre la définition donnée par Tafuri à l'urbanisme corbuséen.

\section{Le conflit des métalangages}

19 L'espace absolu du quadrillage urbanistique ou de la grille cartographique est saisi en surplomb, d'un point de vue qui lui reste extérieur. Il en va autrement pour l'espace pratiqué, qui constitue un milieu conditionnant dont il est difficile de faire abstraction. La sculpture processuelle de Serra fait irruption dans le milieu architecturé ou urbanisé, obligeant ainsi à l'expérimenter de manière contre-intuitive. Elle doit son efficacité à la suppression du socle et aux conséquences ultimes que l'artiste en tire pour critiquer les fonctions assignées au site d'intervention. « Mes œuvres font partie et sont construites dans la structure d'un site, et souvent restructurent, à la fois conceptuellement et perceptuellement, l'organisation de ce site» (Serra, 1993: 40), déclare Serra. Cette référence à la sphère conceptuelle suggère que, par sa morphologie même, la sculpture processuelle contredit les représentations véhiculées par le contexte donné et trouble la manière dont celui-ci demande à être interprété. Au lieu de se limiter à en rehausser les qualités esthétiques ou à en confirmer la raison d'être, elle en subvertit la valeur sociale ou idéologique. L'espace planifié apparaît ainsi comme cadre d'activités mentales, sensitives et motrices qui reflètent le régime actuel de production sociale.

Dans le cas spécifique de Tilted $A \mathrm{Ac}^{13}$, œuvre installée pour quelque temps place de la Fédération à New York, l'égarement venait de la solution de continuité introduite dans la trame urbaine. La place ne remplissait plus sa fonction de raccordement de plusieurs axes de déplacement. Facteur de disjonction de ces axes, l'arc incliné obligeait à emprunter d'autres voies de passage que celles suggérées par la conformation du site. Un contraste se produisait alors entre les anticipations automatiques et l'expérience effective. À défaut d'un point de vue unifiant, le passant était coincé entre la sculpture et les édifices qui entourent la place. L'usage auquel le site est destiné et les comportements qu'il doit implicitement induire se trouvaient contredits, ou plutôt empêchés. Serra souligne le potentiel subversif de sa démarche de la manière suivante :

Fondées sur l'interdépendance du site et de l'œuvre, les sculptures spécifiques au site abordent de manière critique le contenu et le contexte de leur propre site. Une orientation nouvelle du comportement et de la perception à l'égard d'un site requiert une adaptation critique nouvelle du lieu de chacun. [...] Chaque langage a 
sa structure ; on ne peut pas la critiquer si l'on utilise le langage en question. Il faut un autre langage qui traite de la structure du premier et possède une nouvelle structure pour critiquer le premier (Serra, 1990 : 288). celle du métalangage abstrait en lequel consistent l'environnement métropolitain et l'ordre socio-économique qui le détermine. Dérangés par cette anomalie dans le système urbanistique, les usagers, selon la terminologie habituelle, ne peuvent plus performer les tâches considérées comme appropriées au site. L'espace ne saurait être parcouru suivant son ordonnance originelle, ce qui ralentit sensiblement la vitesse de circulation tant recherchée par Le Corbusier et par nombre de planificateurs urbains. Pour en revenir à Tilted Arc, les usagers se trouvaient pris entre deux métalangages dont le conflit les empêchait d'embrasser l'ensemble des constantes et des variables, c'est-à-dire d'adopter le point de vue extérieur, abstraitement objectivant, de la carte ou du plan. Tout cela donnait lieu à des comportements hors norme au sens de non enrégimentés. Ainsi s'expliquent les polémiques suscitées par l'œuvre et son démantèlement définitif au profit d'un réaménagement qui va littéralement dans le sens du lieu.

«Chaque site a sa frontière » (Serra, 1990 : 199), et c'est à partir de la déconstruction de cette frontière à la fois spatiale, comportementale et idéologique que s'opère la convergence entre la sculpture processuelle de Serra et la géographie matérialiste de David Harvey (né en 1935). Harvey conteste à la sociologie urbaine le fait que «l'urbain [y] est considéré comme un objet distinct d'enquête épistémologique » (Harvey, 2001 : 72), pour ensuite pointer la contradiction qui en résulte :

Le développement autonome de la sociologie urbaine repose sur une opposition [...] fondamentale entre la recherche de spécificité dans un horizon restreint et la recherche de généralité dans un univers de discours élargi. [...] En raison de son caractère bourgeois, la sociologie urbaine tend à interpréter les affaires sociales à la lumière d'intérêts et de rôles antagoniques s'intégrant dans une totalité sociétale perçue comme réellement ou potentiellement harmonieuse quant à son fonctionnement (Harvey, $2001:$ 74).

Il est impossible de ne pas reconnaitre dans cette totalité le cosmos auquel Simmel identifie la structure sociale en tant qu'ensemble ordonné d'intérêts individuels et de rôles établis. Harvey vise en effet l'École de Chicago, dont la sociologie simmelienne demeure une des sources fondamentales. «Plus les fragmentations [issues de la division de classe de la métropole] sont profondes, plus la tendance vers la synthèse et la comparaison s'impose » (ibid. : 72), à cause notamment de la «relation [intervertie] entre la structure des problèmes et la structure de la science qui s'en occupe » (Harvey, $2001: 73)$.

Or, nonobstant que la tendance vers la synthèse régisse la planification de la métropole, les fragmentations sous-jacentes peuvent émerger sous forme d'entropie urbaine. L'anarchitecture de Matta-Clark porte sur ce phénomène de "transformation" (entropon, en grec ancien), lequel implique à la fois l'ordre et le désordre. Elle s'élabore au début des années 1970 dans des quartiers de l'île de Manhattan en plein déclin économique suite à la migration de la classe moyenne vers les périphéries et consiste en une série de découpes géométriques pratiquées sur les éléments d'un bâtiment désaffecté ou d'une aire abandonnée. Ces découpes ne peuvent être embrassées dans leur ensemble, ce qui nécessite des déplacements qui s'organisent difficilement en une séquence cohérente. Bien loin de comprendre des parties qui s'intègrent dans un tout 
achevé, l'anarchitecture n'est visible qu'à travers la transgression du double cadre spatial et comportemental fixé par le site. Dans Bronx Floors ${ }^{14}$, elle aboutit déjà à une critique des conceptions rationaliste et fonctionnaliste de l'architectonique, l'entrecroisement des coupes effectuées sur les planchers et sur les murs ayant pour fonction d'entrainer des dislocations au niveau de la «carte mentale " (Matta-Clark, 2011 : 103) du site, dans laquelle Matta-Clark voit le reflet du régime de production en vigueur :

Cette architecture reflète l'iconographie de l'axe occidentale capitaliste. [...] [C]ette tentative monolithique et idéaliste de résoudre les problèmes a non seulement échoué à les résoudre mais a créé des conditions de vie aseptisées et inhumaines au niveau privé et institutionnel. [...] Je perçois mon œuvre comme une scène spéciale, en perpétuelle mutation: un modèle réduit illustrant l'action continue des individus sur l'espace qui les entoure et dans lequel ils se déplacent. Les bâtiments sont des entités fixes, figées, pour la plupart des gens. Le concept d'espace altérable est pratiquement tabou, même chez soi (ibid. : 77 et 79).

L'anarchitecture infirme donc les assomptions sur lesquelles repose l'urbanisme dont elle est contemporaine, assomptions qui se trouvent réunies dans les projets de Le Corbusier et de Hilberseimer. Au mépris de toute délimitation des volumes et des superficies à l'aune de rapports rationnels et inaltérables, Matta-Clark envisage " des formes sans plan - des ordres et des désordres dynamiques " (Matta-Clark, 1973 : sans pagination). Le concept d'espace altérable est aussi celui d'un décalage entre l'exploration concrète et la représentation géométrale de l'espace, lequel s'avère incompatible avec le parallèle postulé par nombre d'architectes et d'urbanistes modernes entre ces deux modes d'expérience. De fait, "si aucun mesurage n'est possible, une conscience plus profonde de l'espace surgit » (Matta-Clark, 1972: sans pagination). L'anarchitecture devient ainsi un "geste métaphorique, sculptural et social» (Matta-Clark, 2011: 100) susceptible de mettre au jour les contradictions que dissimule la tendance vers la synthèse caractéristique de planification et de la sociologie urbaines.

\section{La synthèse urbanistique et ses critiques}

En raison de la solution de continuité qu'elles entraînent dans le tissu de la métropole et dans la chaîne comportementale analogue, les œuvres analysées au paragraphe précédent entrent en résonance aussi avec la critique, menée par Lefebvre, du système actuel de représentation et de production de l'espace. "L'espace d'un ordre se cache dans l'ordre de l'espace » (Lefebvre, 2000 : 332), affirme Lefebvre, et la perte de sens de l'espace urbain se mesure, selon lui, à la convergence de la planification avec une sémiologie qualifiable d'acritique. À la vérité, le planificateur ou le sémiologue qui, sous le signe même de l'idéologie, « conçoit la ville et la réalité urbaine comme système de signes les livre implicitement à la consommation comme objets intégralement consommables : comme valeur d'échange à l'état pur » (Lefebvre, 2009 : 62).

L'hypothèse, émise dans l'introduction à l'aide des remarques de Lefebvre, suivant laquelle le mode d'intégration socio-spatiale adopté par la planification moderne verserait dans l'abstraction trouve enfin confirmation dans le dysfonctionnement que les interventions de Serra et de Matta-Clark provoquent au niveau de la synthèse urbanistique qu'est la métropole. Il y a abstraction et abstraction, comme le rappelle Marx, et l'abstraction fondatrice de la sculpture processuelle et de l'anarchitecture 
dénote un caractère tout à la fois immanent et hétérotopique, tandis que " [l]e contenu de l'espace abstrait consiste en contradictions que la forme abstraite parait réduire mais qu'en réalité son analyse rend manifeste" (Lefebvre, 2000: 332). Chez Le Corbusier et Hilberseimer, ces contradictions sont d'autant plus refoulées que la réduction de l'espace au plan présente deux versants, l'un déductif et l'autre inductif. Il est question, dans les deux cas, de réorganiser la métropole conformément au mode de production sociale isolé par Simmel, ce qui revient à ramener à la surface la structure opératoire de l'urbanisme capitaliste. La dynamique des classes supposée s'enclencher par leurs utopies respectives est en réalité à l'œuvre dès les débuts de l'urbanisation moderne, qui est déjà à elle seule une atopie, attendu qu'en son sein les parcelles de territoire demeurent aussi indéfiniment combinables, subdivisibles, occupables et échangeables que les situations sociales correspondantes.

Ces utopies ne diffèrent qu'en apparence du statu quo, pour ne pas dire qu'elles ne sont que des pseudo-utopies. Peu importe que Le Corbusier envisage une mobilité sociale accrue, la réalisation de son projet ne changerait rien à la « réalité de classe de la ville moderne » (Tafuri, $1979: 105)$. De son côté, bien qu'il propose une alternative socialiste à la planification corbuséenne, Hilberseimer développe lui aussi jusqu'au bout «l'urbanisation de la conscience» (Harvey, $1985: 252)$ que Harvey met directement « en relation avec l'urbanisation du capital » (ibid. : 252). Dans sa tentative de subvertir le modèle urbain calqué sur l'assise capitaliste, Hilberseimer ne réussit pas à se distinguer «des professionnels - ingénieurs, architectes, planificateurs urbains et concepteurs - dont l'objectif consiste à rationaliser les fragments et à imposer une cohérence au système spatial en tant que tout » (Harvey, 1985 : 14). Le rejet final de sa propre tentative indique qu'elle trahit «le concept d'espace homogène, abstrait et objectif » (ibid. : 14-15) auquel est corrélée la méconnaissance non seulement du lien intrinsèque entre l'industrie et le capital, mais aussi du fait que l'urbanisme planifié ne parvient qu'en apparence à fonder une totalité de sens.

Simmel non plus ne va pas au-delà de ce genre d'abstraction, dans la mesure où la découverte de la genèse sociale des catégories a priori de l'individualité, du rôle et de la structure ne le dissuade pas de les considérer comme nécessaires, transhistoriques et transéconomiques. Selon Massimo Cacciari (né en 1944), il « articule une idéologie de la transcendance des conditions générales de la domination sociale capitaliste » (Cacciari, 1993 : 13) sans remettre en question les conditions actuelles de la production sociale et spatiale. Si la métropole «est la forme assumée par le processus de rationalisation des relations sociales " (ibid.: 4), à plus forte raison la rationalisation des relations spatiales parachève ce processus. Simmel a beau constater que, «dans l'évolution de la civilisation moderne, l'accent porte davantage sur ce qu'on peut appeler l'esprit objectif [la production de plus en plus stéréotypée] que sur l'esprit subjectif [l'individuation au niveau du psychisme]» (Simmel, 2007: 38), il ne tire aucune conséquence sociopolitique de ce « résultat de la division croissante du travail» (ibid. : 39), d'où l'objection que lui adresse Cacciari :

Simmel interprète dans un sens idéologique positif tous les rapports économiques et juridiques de la société bourgeoise incarnés dans la métropole : la spiritualité propre à la liberté juridico-formelle de la bourgeoisie est prise par lui pour une véritable liberté - le marché capitaliste pour une intensification de la personnalité et la division sociale du travail pour le fondement d'une égalité individualisée [...]. L'idéologie de la métropole que Simmel expose est donc encore une idéologie de la synthèse. Sa forme englobe à la fois l'émergence de l'individualité extrême dans la 
totalité sociale et l'intégration constante de ce tout dans l'individu (Cacciari, 1993 :

11).

La fonction, que Simmel attribue à la métropole, « de faire place à la querelle et aux tentatives d'unification de ces deux tendances [que sont l'individuation et l'uniformisation] » (Simmel, $2007:$ 43) s'avère donc fallacieuse, l'opposition étant aussi illusoire que la réconciliation sous ces conditions. Pour en revenir aux démarches respectives de Serra et de Matta-Clark, elles montrent à leur tour que l'abstraction auto-organisatrice constitutive de la métropole n'est pas neutre, mais idéologiquement connotée, et que la prétendue transcendance de la planification au sens de Le Corbusier ou de Hilberseimer n'est pas utopique, mais atopique.

\section{BIBLIOGRAPHIE}

CACCIARI M. (1993), Architecture and Nihilism. Philosophy of Modern Architecture, translated by Stephen Sartarelli, New Haven, London, Yale University Press.

CHOAY F. (1972 [1969]), « Sémiologie et urbanisme », in Choay F., Banham R., Baird G. et al., Le sens de la ville, Paris, Seuil, pp. 9-30.

FARINELLI F. (2009 [2003]), De la raison cartographique, trad. par Katia Bienvenu et Brice Gruet, Paris, Éditions du Comité des travaux historiques et scientifiques,.

FISHMAN R. (1979 [1977]), L'utopie urbaine au XXe siècle. Ebenezer Howard, Frank Lloyd Wright, Le Corbusier [1977], Bruxelles, Pierre Mardaga.

FOUCAULT M., (2004 [1967]), « Des espaces autres », Empan, 2, no. 54, pp. 12-19.

HARVEY D. (2001), Spaces of Capital. Towards a Critical Geography, New York, Routledge.

HARVEY D. (1985), Consciousness and the Urban Experience. Studies in the History and Theory of Capitalist Urbanization, Baltimore, John Hopkins University Press.

HILBERSEIMER L. (2012 [1927]), Metropolisarchitecture and Selected Essays, translated by Richard Anderson, New York, Columbia GSAPP.

HILBERSEIMER L. (1988 [1963]), extrait de « Entfaltung einer Planungsidee » cité in Pommer R., «"More a Necropolis than a Metropolis". Ludwig Hilberseimer's Highrise City and Modern City Planning ", in Pommer R. (dir.), In the Shadow of Mies. Ludwig Hilberseimer. Architect, Educator, and Urban Planner, New York, The Art Institute of Chicago and Rizzoli International Publications, inc. pp. 16-53.

JONAS S. (2008), « Simmel et Berlin. De la Grande ville à la Métropole », in Füzesséry S., Simay P. (dir.), Le choc des métropoles. Simmel, Kracauer, Benjamin, Paris, Tel-Aviv, Éditions de l'éclat, pp. 55-80.

LE CORBUSIER (1928 [1923]), Vers une architecture, Paris, Georges Crès et C ${ }^{\text {ie }}$.

LE CORBUSIER (1925), Urbanisme, Paris, Georges Crès et $C^{\text {ie }}$.

LEFEBVRE H. (2009 [1968]), Le droit à la ville, Paris, Economica-Anthropos. 
LEFEBVRE H. (2000 [1974]), La Production de l'espace, Paris, Anthropos.

MARX K, (1980 [1857]), Manuscrits de 1857-1858 (« Grundrisse »), t. I, Paris, Éditions sociales.

MATTA-CLARK G. (2011), Entretiens, trad. par Raphaëlle Brin, Paris, Lutanie.

MATTA-CLARK G. (1972-1973), Cahier de notes, propriété de Gordon Marra-Clark en dépôt au Centre canadien d'architecture, Montréal, sans pagination.

POMMER R. (1988), « “More a Necropolis than a Metropolis”. Ludwig Hilberseimer's Highrise City and Modern City Planning ", in Pommer R. (dir.), In the Shadow of Mies. Ludwig Hilberseimer. Architect, Educator, and Urban Planner, New York, The Art Institute of Chicago and Rizzoli International Publications, inc., pp. 16-53.

SIMMEL G. (2007 [1903]), Les grandes villes et la vie de l'esprit, suivi de Pont et porte, trad. par Françoise Ferlan, Paris, L'Herne.

SIMMEL G. (1999 [1908]), Sociologie. Études sur les formes de la socialisation, trad. par Lilyane Deroche-Gurcel et Sibylle Muller, Paris, Presses Universitaires de France.

SIMMEL G. (1981 [1917]), Sociologie et épistémologie, trad. par Liliane Gasparini, Paris, Presses Universitaires de France,.

SITTE C. (1980 [1889]), L'art de bâtir les villes. L'urbanisme selon ses fondements artistiques, trad. par David Wieczorek, Paris, L’Équerre.

SERRA R. (1993), cité in Pacquement A., Richard Serra, Paris, Éditions du Centre Pompidou.

SERRA R. (1990), Écrits et Entretiens [1970-1989], trad. par Gilles Courtois, Paris, Lelong.

TAFURI M. (1979 [1973]), Projet et utopie. De l'Avant-garde à la Métropole, trad. par Françoise Brun, adaptée par Ligia Ravé-Emy, Paris, Dunod.

WIGLEY M. (1988), « Deconstructivist Architecture », in Johnson P., Wigley M. (dir.),

Deconstructivist Architecture, London, Thames \& Hudson, pp. 10-20.

\section{NOTES}

1. Ces principes communs apparaissent aussitôt à la lecture des écrits de Simmel sur la métropole, tous consacrés à Berlin. Certaines formulations sociologiques «peuvent être considérées comme des approches fonctionnelles de la métropole : la métropole se définit comme un réseau; la grande ville a toujours été le siège de l'économie monétaire; la métropole se nourrit de la production destinée au marché ; la métropole est le principal centre de l'échange monétaire" (Jonas, 2008 : 61). D'autres «nous renvoient à l'esprit (Geist) de la métropole : la grande ville est le siège de la plus haute division du travail ; la métropole a toujours été un centre du cosmopolitisme; la grande ville est le siège de la liberté personnelle ; elle suggère le penchant à l'existence personnelle la plus individuelle; elle est la scène réelle de la culture objective ; la métropole est un modèle moderne du schéma temporel supra-subjectif » (Jonas, $2008: 61$ ).

2. «Au stade final de l'industrie, de l'échange et de la circulation - au degré le plus élevé d'organisation sociale actuellement connu - apparaissent la métropole et la ville globale. La métropole est produite par le développement de l'économie moderne. Les grandes villes du passé diffèrent de la métropole en raison même de leurs différentes assises économiques.» (Hilberseimer, 2012 : 84-85.)

3. Simmel précise que "ces modifications et ces transformations, qui empêchent sa [de l'individu] connaissance idéale, sont justement les conditions qui rendent possibles les seules relations que nous connaissons comme sociales - à peu près comme, chez Kant, seules les 
catégories de l'entendement, qui modèlent le donné immédiat pour constituer des objets tout à fait nouveaux, font du monde donné un monde connaissable » (Simmel, 1999 : 70).

4. «La société produit peut-être ainsi l'élaboration la plus consciente, du moins la plus universelle, d'une forme fondamentale de la vie : l'âme individuelle ne peut jamais se retrouver à l'intérieur d'une relation si elle n'est pas en même temps à l'extérieur de celle-ci, elle ne fait pas partie intégrante d'un ordre sans être en même temps face à lui. Cela va des ensembles les plus transcendants et universels jusqu'aux plus singuliers et contingents. » (Simmel, 1999 : 72-73.)

5. «D'une manière générale, cette image de la société a une analogie, comme en miniature, infiniment simplifiée et en quelque sorte stylisée avec la bureaucratie : en tant que telle, elle est composée d'un certain ordre de "positions", de tâches prédéterminées, qui, détachées de leurs différents vecteurs, constituent une structure idéale; à l'intérieur de celle-ci, chacun des nouveaux arrivants trouve une place clairement déterminée, qui l'attendait en quelque sorte et avec laquelle ses énergies doivent s'harmoniser. » (Simmel, 1999 : 76. )

6. Le Corbusier (1922), Ville contemporaine de trois millions d'habitants, sans lieu, projet non réalisé, encre et aquarelle sur papier, dimensions variables, Fondation Le Corbusier, Paris.

7. Voir Sitte, $1980: 99$ et 106.

8. Le Corbusier (1925), Plan Voisin, Paris, projet non réalisé, encre et aquarelle sur papier, dimensions variables, Fondation Le Corbusier, Paris.

9. «La Ville contemporaine de Le Corbusier était, au vingtième siècle, l'incarnation de la Société que [Henri de] Saint-Simon avait prévue au dix-neuvième. Là, selon la formule fameuse de SaintSimon, l'“administration des biens" avait remplacé "le gouvernement des hommes". Dans la Ville contemporaine les structures gouvernementales étaient littéralement éclipsées. Elles étaient situées sur la frange du centre des affaires, à l'ombre des grandes tours. Ces tours étaient le quartier général de l'élite de Le Corbusier ; chefs d'entreprises, savants et artistes, exactement les trois mêmes catégories qui constituaient les industriels de Saint-Simon. Comme ces industriels, l'élite de Le Corbusier devait comprendre les membres les plus doués de la population active. Ils apporteraient à la société, grâce à leur action bénéfique, l'administration, la prospérité, l'ordre et la beauté. » (Fishman, 1979 : 148-149.)

10. «Le chaos de la métropole moderne ne peut être affronté qu'avec des expérimentations dans le domaine de la démonstration théorétique. L'objectif est de développer, dans l'abstrait et en réponse aux exigences contemporaines, les principes fondamentaux de la planification urbaine. Cette démarche doit aboutir à la production de règles générales permettant de résoudre certains problèmes concrets. » (Hilberseimer, 2012 : 112.)

11. Hilberseimer L. (1928), Projet de développement de Berlin, Berlin, projet non réalisé, encre sur papier collé sur photographie aérienne, 17,2 x $25 \mathrm{~cm}$, Art Institute of Chicago, Chicago.

12. Hilberseimer L. (1924), Ville verticale, sans lieu, projet non réalisé, encre et aquarelle sur papier, dimensions variables, Art Institute of Chicago, Chicago.

13. Serra R. (1981), Tilted Arc, œuvre démantelée en 1989, section de cylindre, acier imperméable, $366 \mathrm{~cm}$ x 36,58 m x 6,5 cm, Federal Plaza, New York.

14. Matta-Clark G. (1972-1973), Bronx Floors, Bronx, interventions in situ, découpes et tirage sur papier d'après négatifs chromogènes, dimensions variables, Estate of Gordon Matta-Clark, New York. 


\title{
RÉSUMÉS
}

L'enjeu du présent article est de discuter deux conceptions antagonistes de la relation réciproque que la socialisation entretient avec la spatialisation. Deux conceptions auxquelles renvoient les expressions "production sociale " et "production de l'espace ", empruntées respectivement à Georg Simmel et à Henri Lefebvre. L'antagonisme relève, en l'occurrence, de la fonction assignée à la géométrie en tant qu'abstraction socio-spatiale, y compris dans le domaine de l'urbanisme. Des architectes modernistes comme Le Corbusier et Ludwig Hilberseimer pensent, en effet, que les rapports géométriquement établis dans le cadre de leurs projets d'aménagement respectifs permettent de synthétiser les principaux facteurs de production socio-spatiale. Sous cet aspect, ils s'inscrivent dans le sillage de Simmel, dont la déduction empirique de catégories telles que l'individualité, le rôle et la structure constitue déjà une abstraction typologique, voire une géométrie sociale. Or, en raison de la solution de continuité qu'elles introduisent dans le tissu de la métropole capitaliste, les sculptures de Richard Serra et les anarchitectures de Gordon MattaClark entrent plutôt en résonance avec l'analyse, menée par Lefebvre, de la production sociospatiale actuelle. Critiques paradoxales de la géométrisation au moyen de formes géométriques, elles mettent simultanément au jour et en cause le substrat idéologique de la planification moderne, nous permettant ainsi de discuter deux régimes d'abstraction tout aussi antagonistes.

The aim of this article is to discuss two antagonistic conceptions of the relationship between socialization and spatialization. These are the conceptions referred to by the expressions "social production" and "production of space" borrowed, respectively, from Georg Simmel and Henri Lefebvre. The antagonism is rooted, in this case, on the function assigned to geometry as sociospatial abstraction, particularly in the field of urban planning. Modernist architects like Le Corbusier and Ludwig Hilberseimer consider that the geometrically established relationships within the framework of their respective development projects make it possible to synthesize the main factors of socio-spatial production. In this respect, they follow Simmel, whose empirical deduction of categories such as individuality, role and structure is already a typological abstraction, or even a social geometry. As opposed to that, considering the way they interrupt the continuous fabric of the capitalist metropolis, Richard Serra's sculptures and Gordon MattaClark's anarchitectures rather resonate with Lefebvre's analysis of the current socio-spatial production. These works are paradoxical critiques of geometrization by means of geometrical shapes. More precisely, they uncover and question the ideological substratum of modern planning, thus allowing us to discuss two equally antagonistic regimes of abstraction.

\section{INDEX}

Mots-clés : abstraction, socialisation, sociologie, spatialisation, urbanisme

Keywords : Abstraction, Socialization, Sociology, Spatialization, Urbanism

\author{
AUTEUR \\ DIEGO SCALCO \\ Docteur en philosophie, chercheur à l'Institut « Arts, créations, théories et esthétiques » (ACTE), \\ ancien Attaché temporaire d'enseignement et de recherche (ATER) à l'Université Paris 1 \\ (Panthéon-Sorbonne), Diego Scalco enseigne actuellement la philosophie de l'art dans le même
}


établissement, l'esthétique et la théorie des images à l'Université Gustave Eiffel et l'esthétique à l'Université de Picardie - Jules Verne.

diegoscalco[at]hotmail.com 